\title{
P-MAPPING SPACES FOR P-OPERATOR SPACES ON $L_{p}$ SPACES
}

\author{
Y. F. ZHAO AND Z. DONG
}

\begin{abstract}
In this paper, we introduce p-mapping spaces for p-operator spaces on $L_{p}$ spaces, which can be regarded as pgeneralization of mapping spaces for operator spaces. We then apply p-mapping spaces to study the p-local reflexivity for poperator spaces on $L_{p}$ spaces.
\end{abstract}

\section{Introduction}

Throughout this writing, we always assume $1<p<\infty$ unless stated otherwise. Given $p$, its conjugate exponent is denoted by $p^{\prime}$ so that $1 / p+1 / p^{\prime}=1$. Some fundamental results (p-completely bounded maps, p-Haagerup and pprojective tensor products) for p-operator spaces have been studied by Pisier [13], Le Merdy [9], and Daws [2]. In [1], the p-injective tensor product was introduced for p-operator spaces, and various properties related to this tensor product were studied, including the p-approximation property for p-operator spaces on $L_{p}$ spaces. The p-operator space tensor products are crucial in this paper.

In Section 2, we recall some basic notations and properties of p-operator spaces developed by Le Merdy [9] and Daws [2]. Obviously, certain operator space properties may fail for general p-operator spaces. For instance, there is non-existence of the corresponding Arveson-Wittstock-Hahn-Banach theorem for p-completely bounded maps (see Lee [12]). The p-Haagerup tensor product for p-operator spaces is not injective anymore (see Le Merdy [9]).

The theory of mapping spaces for operator spaces arose from [5], [6], [7], [4] and [8]. The most successful application of mapping spaces in operator spaces is to show that the dual of every $C^{*}$-algebra is locally reflexive

Received December 19, 2014; received in final form April 1, 2015.

Partially supported by the National Natural Science Foundation of China (No. 11271321) and the Fundamental Research Funds for the Central Universities.

2010 Mathematics Subject Classification. 46L06, 46L07. 
in [4]. We first in Section 3 introduce a p-complete isometry $T_{n}(V) \cong T_{n} \stackrel{\wedge_{p}}{\otimes} V$. The analysis of p-completely 1-summing mappings rests upon a careful study of $T_{n}(V)$. Here we respectively explore the p-completely nuclear mappings in Section 3, the p-completely integral mappings in Section 4 and the pcompletely 1 -summing and $\infty$-summing mappings in Section 5 , for p-operator spaces on $L_{p}$ spaces. In Section 6 , we then apply these p-mapping spaces to the study of the p-local reflexivity for p-operator spaces on $L_{p}$ spaces. We prove in Theorem 6.2 the equivalence with the isometric conditions. However, due to the lack of the corresponding Arveson-Wittstock-Hahn-Banach theorem for p-completely bounded maps, it is not clear whether this is true for the p-completely isometric conditions. Finally, we end the section by an observation on p-completely 1 -summing and $\infty$-summing mappings in the condition of the p-local reflexivity.

\section{P-operator spaces}

Let $1<p<\infty$. A p-operator space is a Banach space $V$ together with a matrix norm, that is, a norm $\|\cdot\|_{n}$ on each matrix space $M_{n}(V)$, which satisfies the following two conditions $\mathcal{D}_{\infty}:\|x \oplus y\|_{n+m}=\max \left\{\|x\|_{n},\|y\|_{m}\right\}$ for $x \in M_{n}(V)$ and $y \in M_{m}(V), \mathcal{M}_{p}:\|\alpha x \beta\|_{n} \leq\|\alpha\|\|x\|_{n}\|\beta\|$ for $x \in M_{n}(V)$ and $\alpha, \beta \in M_{n}=B\left(l_{p}^{n}\right)$.

When $V$ is a p-operator subspace of some $B\left(L_{p}(\mu)\right)$, then we say that $V$ is a p-operator space on $L_{p}$ space. Unlike operator spaces, there exists a poperator space $V$ such that the inclusion $\kappa_{V}: V \rightarrow V^{* *}$ is not p-completely isometric (see Daws [2]). By Proposition 4.9 in [2], however, $\kappa_{V}$ is a p-complete isometry if and only if $V$ is a p-operator space on $L_{p}$ space.

In [2], Daws defined and studied the p-projective tensor product. The p-projective tensor product preserves most of properties of operator space projective tensor product. For instance, the tensor product of p-complete contractions (respectively, p-complete quotients) is again a p-complete contraction (respectively, a p-complete quotient). The p-projective tensor product is associative, that is, $\left(V \stackrel{\wedge_{p}}{\otimes} W\right) \stackrel{\wedge_{p}}{\otimes} Z=V \stackrel{\wedge_{p}}{\otimes}\left(W \stackrel{\wedge_{p}}{\otimes} Z\right)$, and commutative, that is, $V \stackrel{\wedge_{p}}{\otimes} W=W \stackrel{\wedge_{p}}{\otimes} V$. We also have the p-completely isometric identifications

$$
\mathrm{CB}_{p}\left(X \stackrel{\wedge_{p}}{\otimes} Y, Z\right)=\mathrm{CB}_{p}(X \times Y, Z)=\mathrm{CB}_{p}\left(X, \mathrm{CB}_{p}(Y, Z)\right)
$$

In particular,

$$
\left(X \stackrel{\wedge_{p}}{\otimes} Y\right)^{*}=\mathrm{CB}_{p}\left(X, Y^{*}\right) .
$$

In [1], the authors introduced the p-injective tensor product. The tensor product of $\mathrm{p}$-complete contractions under the $\mathrm{p}$-injective tensor product is again a p-complete contraction. In particular, if $V$ and $W$ are p-operator 
spaces, the bilinear mapping

$$
V \times W \rightarrow V \otimes_{\vee_{p}} W:(v, w) \mapsto v \otimes w
$$

is p-completely contractive, and thus determines a p-complete contraction

$$
\Phi: V \stackrel{\wedge_{p}}{\otimes} W \rightarrow V \stackrel{\vee_{p}}{\otimes} W .
$$

Let $V, W$ be p-operator spaces on $L_{p}$ spaces. It was known from [1] that for each $u \in M_{n}(V \otimes W)$, the p-injective tensor norm $\|u\|_{\vee_{p}}$ can be expressed by

$$
\|u\|_{\vee_{p}}=\sup \left\{\left\|(\varphi \otimes \psi)_{n}(u)\right\|: \varphi \in M_{m}\left(V^{*}\right)_{1}, \psi \in M_{k}\left(W^{*}\right)_{1}, m, k \in \mathbb{N}\right\} .
$$

If $V \subseteq B\left(L_{p}(\mu)\right)$, then we have a p-completely isometric isomorphism

$$
M_{n}(V)=M_{n} \stackrel{\vee_{p}}{\otimes} V
$$

Let $V, W$ be p-operator spaces on $L_{p}$ spaces, then the canonical inclusion

$$
V^{*} \stackrel{\vee_{p}}{\otimes} W \hookrightarrow \mathrm{CB}_{p}(V, W)
$$

is a p-completely isometric injection. We do not know whether the p-injective tensor product is injective. But if all p-operator spaces under consideration are on $L_{p}$ spaces, then the p-injective tensor product is injective (see [11]).

THEOREM 2.1. Suppose that $V, W$, and $X$ are $p$-operator spaces. Then the natural mappings

$$
V \otimes_{\wedge_{p}}\left(W \otimes_{\vee_{p}} X\right) \rightarrow\left(V \otimes_{\wedge_{p}} W\right) \otimes_{\vee_{p}} X
$$

are $p$-completely contractive.

Proof. we let $Z=W \otimes \vee_{p} X$. Given $u \in M_{n}(V \otimes Z)$ and $\varepsilon>0$, we may assume that

$$
u=\alpha(v \otimes z) \beta=\left[\sum_{i, j, k, l} \alpha_{g,(i, k)}\left(v_{i j} \otimes z_{k l}\right) \beta_{(j, l), h}\right],
$$

where $v \in M_{r}(Z), z \in M_{q}(Z), \alpha \in M_{n, r \times q}$, and $\beta \in M_{r \times q, n}$ satisfy

$$
\|\alpha\|\|v\|\|z\|_{\vee_{p}}\|\beta\|<\|u\|_{\wedge_{p}}+\varepsilon
$$

We let $z=\left[z_{k l}\right]$, where

$$
z_{k l}=\sum_{t} w_{k l}^{(t)} \otimes x_{k l}^{(t)}
$$

with $w_{k l}^{(t)} \in W$ and $x_{k l}^{(t)} \in X$. Then we have

$$
u=\left[\sum_{i, j, k, l, t} \alpha_{g,(i, k)}\left(\left(v_{i j} \otimes w_{k l}^{(t)}\right) \otimes x_{k l}^{(t)}\right) \beta_{(j, l), h}\right] .
$$

If $\|v\|=0$, it is easy see that

$$
\|u\|_{V \otimes_{\wedge_{p}}\left(W \otimes_{\vee_{p}} X\right)}=\|u\|_{\left(V \otimes_{\wedge_{p}} W\right) \otimes_{\vee_{p}} X}
$$


So here, we can assume $\|v\|>0$.

From the definition of p-operator space injective tensor product norm in [11],

$$
\begin{aligned}
& \|u\|_{\left(V \otimes \wedge_{p} W\right) \otimes \vee_{p} X} \\
& =\sup \left\{\left\|\left[\sum_{i, j, k, l, t} \alpha_{g,(i, k)} e_{s t}\left(v_{i j} \otimes w_{k l}^{(t)}\right) x_{k l}^{(t)} \beta_{(j, l), h}\right]\right\|_{M_{m n}(X)}:\right. \\
& \left.\quad m \in \mathbb{N}, e=\left[e_{s t}\right] \in M_{m}\left(\left(V \otimes_{\wedge_{p}} W\right)^{*}\right)_{1}\right\},
\end{aligned}
$$

where $M_{m}\left(\left(V \otimes_{\wedge_{p}} W\right)^{*}\right)_{1}$ denotes the closed unit ball of

$$
M_{m}\left(\left(V \otimes_{\wedge_{p}} W\right)^{*}\right)=\mathrm{CB}_{p}\left(\left(V \otimes_{\wedge_{p}} W\right), M_{m}\right) .
$$

If we fix such element $e, e$ determines a p-complete contraction

$$
E \in \mathrm{CB}_{p}\left(V, \mathrm{CB}_{p}\left(W, M_{m}\right)\right),
$$

where

$$
E\left(v_{0}\right)\left(w_{0}\right)=e\left(v_{0} \otimes w_{0}\right)
$$

for any $v_{0} \in V$ and $w_{0} \in W$. Thus, if $f_{i j}=E\left(v_{i j}\right) /\|v\|$, then

$$
f=\left[f_{i j}\right] \in M_{r}\left(\mathrm{CB}_{p}\left(W, M_{m}\right)\right)=\mathrm{CB}_{p}\left(W, M_{r \times m}\right)
$$

satisfies

$$
\|f\|_{p c b} \leq 1
$$

So we have

$$
\begin{aligned}
& \left\|\left[\sum_{i, j, k, l, t} \alpha_{g,(i, k)} e_{s t}\left(v_{i j} \otimes w_{k l}^{(t)}\right) x_{k l}^{(t)} \beta_{(j, l), h}\right]\right\|_{M_{m n}(X)} \\
& =\left\|\left[\sum_{i, j, k, l, t} \alpha_{g,(i, k)} E\left(v_{i j}\right)\left(w_{k l}^{(t)}\right) x_{k l}^{(t)} \beta_{(j, l), h}\right]\right\| \\
& =\left\|\left[\sum_{i, j, k, l} \alpha_{g,(i, k)}\left(\sum_{t} f_{i j}\left(w_{k l}^{(t)}\right) x_{k l}^{(t)}\right) \beta_{(j, l), h}\right]\right\|\|v\| \\
& \quad \leq\|\alpha\|\|z\|_{\vee_{p}}\|\beta\|\|v\| \\
& \quad<\|u\|_{\wedge_{p}}+\varepsilon .
\end{aligned}
$$

It follows that

$$
\|u\|_{\left(V \otimes_{\wedge_{p}} W\right) \otimes \vee_{p} X} \leq\|u\|_{V \otimes_{\wedge_{p}}\left(W \otimes_{\vee_{p}} X\right)} .
$$

Thus we obtain the desired inequality.

TheOrem 2.2. Let $V, W$, and $X$ be p-operator spaces on $L_{p}$ spaces. Then we have the p-completely isometric isomorphisms

$$
V \stackrel{\vee_{p}}{\otimes} W \cong W \stackrel{\vee_{p}}{\otimes} V
$$


and

$$
\left(V \stackrel{\vee_{p}}{\otimes} W\right) \stackrel{\vee_{p}}{\otimes} X \cong V \stackrel{\vee_{p}}{\otimes}\left(W \stackrel{\vee_{p}}{\otimes} X\right)
$$

Proof. Given any index set $I, J$, and $K$, we have the natural isometries

$$
\left(l_{p}(I) \otimes_{p} l_{p}(J)\right) \otimes_{p} l_{p}(K) \cong l_{p}(I) \otimes_{p}\left(l_{p}(J) \otimes_{p} l_{p}(K)\right)
$$

and

$$
l_{p}(I) \otimes_{p} l_{p}(J) \cong l_{p}(J) \otimes_{p} l_{p}(I) .
$$

Thus, the results follow from Proposition 3.3 in [1].

THEOREM 2.3. Let $V, W$ be p-operator spaces on $L_{p}$ spaces with $V$ or $W$ finite-dimensional. Then we have the p-complete isometry

$$
V^{*} \stackrel{\vee_{p}}{\otimes} W \cong \mathrm{CB}_{p}(V, W)
$$

Proof. We have the p-completely isometric inclusion

$$
V^{*} \stackrel{\vee_{p}}{\otimes} W \hookrightarrow \mathrm{CB}_{p}(V, W) .
$$

Hence to prove the identification, it suffices to show

$$
\varphi: V^{*} \stackrel{\vee_{p}}{\otimes} W \hookrightarrow \mathrm{CB}_{p}(V, W)
$$

is surjective. Since $V$ or $W$ is finite-dimensional, we have the identification $V^{*} \otimes W \cong \operatorname{FCB}_{p}(V, W)$. Thus, we obtain that $\varphi$ is surjective.

\section{P-completely nuclear mappings}

Definition 3.1. Let $V, W, U, X$ be p-operator spaces on $L_{p}$ spaces. A poperator space mapping ideal $\mathcal{O}$ is an assignment to each pair of p-operator spaces $V, W$ of a linear space $\mathcal{O}$ of p-completely bounded mappings $\varphi: V \rightarrow W$, together with a p-operator space matrix norm $\|\cdot\|_{\mathcal{O}}$, such that for each $\varphi \in M_{n}(\mathcal{O})$,

(a) $\|\varphi\|_{p c b} \leq\|\varphi\|_{\mathcal{O}}$ and

(b) for any linear mappings $r: U \rightarrow V$ and $s: W \rightarrow X$,

$$
\left\|s_{n} \circ \varphi \circ r\right\|_{\mathcal{O}} \leq\|s\|_{p c b}\|\varphi\|_{\mathcal{O}}\|r\|_{p c b} .
$$

We say the p-operator space mapping ideal $\mathcal{O}$ is local if for each linear mapping $\varphi: V \rightarrow W$

$$
\|\varphi\|_{\mathcal{O}}=\sup \left\{\left\|\left.\varphi\right|_{L}\right\|_{\mathcal{O}} \text { : for any finite-dimensional subspace } L \subseteq V\right\} \text {. }
$$

Definition 3.2. Let $V, W$ be p-operator spaces on $L_{p}$ spaces. Guided by operator spaces, we define the p-completely nuclear mappings $\mathcal{N}_{p}(V, W)$ to be the image of the mapping

$$
\Phi: V^{*} \stackrel{\wedge_{p}}{\otimes} W \rightarrow V^{*} \stackrel{\vee_{p}}{\otimes} W \subseteq \mathrm{CB}_{p}(V, W)
$$


with the quotient p-operator space structure determined by the identification

$$
\mathcal{N}_{p}(V, W) \cong \frac{V^{*} \stackrel{\wedge_{p}}{\otimes} W}{\operatorname{ker} \Phi} .
$$

Let $\nu_{n}^{p}$ be the matrix norm on $M_{n}\left(\mathcal{N}_{p}(V, W)\right)$.

For exploring the identifications in the p-completely nuclear mappings, we define the following spaces with a norm similar to $\|\cdot\|_{1}$ in operator space theory, which has been introduced by Lee [10].

Definition 3.3. For a p-operator space $V$, let $T_{n}(V)$ denote a Banach space

$$
\left(M_{n}(V),\|\cdot\|_{1, n}\right)
$$

where $\|\cdot\|_{1, n}$ is defined by

$$
\begin{gathered}
\|v\|_{1, n}=\inf \left\{\|\alpha\|_{p^{\prime}}\|w\|\|\beta\|_{p}: r \in \mathbb{N}, v=\alpha w \beta,\right. \\
\left.\alpha \in M_{n, r}, \beta \in M_{r, n}, w \in M_{r}(V)\right\},
\end{gathered}
$$

where $\|\alpha\|_{p^{\prime}}=\left(\sum_{i=1}^{n} \sum_{j=1}^{r}\left|\alpha_{i j}\right|^{p^{\prime}}\right)^{1 / p^{\prime}}$ and $\|\beta\|_{p}=\left(\sum_{k=1}^{r} \sum_{l=1}^{n}\left|\beta_{k l}\right|^{p}\right)^{1 / p}$.

For a p-operator space $V, T_{n}(V)^{*} \cong M_{n}\left(V^{*}\right) \cong \mathrm{CB}_{p}\left(V, M_{n}\right)$ are isometric isomorphisms ([11], Lemma 3.4). Also, these identifications are p-completely isometric isomorphisms. Let nuclear operators $\mathcal{N}\left(l_{p}^{n}\right)$ to be the image of the mapping

$$
\Phi:\left(l_{p}^{n}\right)^{*} \stackrel{\wedge_{p}}{\otimes} l_{p}^{n} \rightarrow\left(l_{p}^{n}\right)^{*} \stackrel{\vee_{p}}{\otimes} l_{p}^{n} \subseteq B\left(l_{p}^{n}\right)
$$

with the quotient norm coming from $\mathcal{N}\left(l_{p}^{n}\right) \cong \frac{\left(l_{p}^{n}\right)^{*} \wedge_{p} \otimes l_{p}^{n}}{\operatorname{ker} \Phi}$. If we use

$$
T_{n} \cong \mathcal{N}\left(l_{p}^{n}\right), \quad M_{n}(\mathbb{C}) \cong B\left(l_{p}^{n}\right),
$$

then by Proposition 2.2 in [1], we have

$$
T_{n} \cong M_{n}^{*}, \quad M_{n} \cong T_{n}^{*},
$$

and

$$
T_{\infty} \cong K_{\infty}^{*}, \quad M_{\infty} \cong T_{\infty}^{*} .
$$

Let $V$ be a p-operator space on $L_{p}$ space. By Theorem 3.6 in [1], we have the isometric isomorphism

$$
\left(M_{n} \stackrel{\vee_{p}}{\otimes} V\right)^{*} \cong T_{n} \stackrel{\wedge_{p}}{\otimes} V^{*} .
$$

LEMmA 3.4. Let $V, W$ be p-operator spaces on $L_{p}$ spaces. Given linear mappings $\varphi_{n}: M_{n}(V) \rightarrow M_{n}(W)$ and $T_{n}(\varphi): T_{n}(V) \rightarrow T_{n}(W)$ for each $n \in \mathbb{N}$. If $T_{n}(\varphi)$ is an isometric injection for each $n \in \mathbb{N}$, then so is $\varphi_{n}$. 
Proof. We may prove that if $\varphi_{n}$ is a quotient mapping for each $n \in \mathbb{N}$, then so is $T_{n}(\varphi)$. Let us suppose that $\varphi_{n}$ is a quotient mapping for each $n \in \mathbb{N}$. For any $w \in T_{n}(W)$ with $\|w\|_{1, n}<1$, we may assume that $w=\alpha \widetilde{w} \beta$, where $\widetilde{w} \in M_{r}(W), \alpha \in M_{n, r}$ and $\beta \in M_{r, n}$ satisfy $\|\widetilde{w}\|,\|\alpha\|_{p^{\prime}},\|\beta\|_{p}<1$. By hypothesis, we may choose an element $\widetilde{v} \in M_{r}(V)$ with $\|\widetilde{v}\|<1$, for which $\varphi_{r}(\widetilde{v})=\widetilde{w}$. If we let $v=\alpha \widetilde{v} \beta$, then it follows that $\|v\|_{1, n}<1$ and $T_{n}(\varphi)(v)=w$. So $T_{n}(\varphi)$ is a quotient mapping for each $n \in \mathbb{N}$.

We have the isometric isomorphisms

$$
M_{n}(V)^{*} \cong\left(M_{n} \stackrel{\vee_{p}}{\otimes} V\right)^{*} \cong T_{n} \stackrel{\wedge_{p}}{\otimes} V^{*} \cong T_{n}\left(V^{*}\right) .
$$

We can note that $T_{n}(\varphi)^{*}=\left(\varphi^{*}\right)_{n}$ and $\left(\varphi_{n}\right)^{*}=T_{n}\left(\varphi^{*}\right)$. Thus from (A.2.1) in [8], $T_{n}(\varphi)$ is an isometric injection for each $n \in \mathbb{N} \Rightarrow\left(\varphi^{*}\right)_{n}$ is a quotient mapping for each $n \in \mathbb{N} \Rightarrow T_{n}\left(\varphi^{*}\right)$ is a quotient mapping for each $n \in \mathbb{N} \Rightarrow \varphi_{n}$ is an isometric injection for each $n \in \mathbb{N}$.

For any p-operator space $V$, we have the p-complete isometries

$$
\left(T_{n} \stackrel{\wedge_{p}}{\otimes} V\right)^{*} \cong \mathrm{CB}_{p}\left(V, M_{n}\right) \cong M_{n}\left(V^{*}\right) \cong\left(T_{n}(V)\right)^{*} .
$$

Then, we obtain a natural isometry $T_{n}(V) \cong T_{n} \stackrel{\wedge_{p}}{\otimes} V$.

COROLlaRY 3.5. Let $V$ be a p-operator space. The natural isometry $T_{n}(V) \cong T_{n} \stackrel{\wedge_{p}}{\otimes} V$ is a p-completely isometric isomorphism.

Proof. We have the p-complete isometries

$$
\left(T_{n} \stackrel{\wedge_{p}}{\otimes} V\right)^{*} \cong \mathrm{CB}_{p}\left(V, M_{n}\right) \cong M_{n}\left(V^{*}\right) \cong\left(T_{n}(V)\right)^{*} .
$$

Then for each $r \in \mathbb{N}$, we have the isometries

$$
\left(T_{r}\left(T_{n} \stackrel{\wedge_{p}}{\otimes} V\right)\right)^{*} \cong M_{r}\left(\left(T_{n} \stackrel{\wedge_{p}}{\otimes} V\right)^{*}\right) \cong M_{r}\left(\left(T_{n}(V)\right)^{*}\right) \cong\left(T_{r}\left(T_{n}(V)\right)\right)^{*},
$$

and thus $T_{r}\left(T_{n} \stackrel{\wedge_{p}}{\otimes} V\right) \cong T_{r}\left(T_{n}(V)\right)$. From Lemma 3.4, for each $r \in \mathbb{N}$ we have the isometry $M_{r}\left(T_{n} \stackrel{\wedge_{p}}{\otimes} V\right) \cong M_{r}\left(T_{n}(V)\right)$. Then, $T_{n}(V) \cong T_{n} \stackrel{\wedge_{p}}{\otimes} V$ is a p-completely isometric isomorphism.

THEOREM 3.6. Let $V$ be a p-operator space on $L_{p}$ space. We have the following p-completely isometric identifications

$$
M_{n}(V)^{* *} \cong M_{n}\left(V^{* *}\right)
$$

and

$$
M_{n}(V)^{*} \cong T_{n}\left(V^{*}\right)
$$


Proof. We have the isometric isomorphisms

$$
M_{n}(V)^{*} \cong\left(M_{n} \stackrel{\vee_{p}}{\otimes} V\right)^{*} \cong T_{n} \stackrel{\wedge_{p}}{\otimes} V^{*} \cong T_{n}\left(V^{*}\right)
$$

It is easy to see that $M_{n}(V)^{* *} \cong M_{n}\left(V^{* *}\right)$ is a p-completely isometric isomorphism. Then we just need to show that

$$
M_{r}\left(T_{n}\left(V^{*}\right)\right) \rightarrow M_{r}\left(M_{n}(V)^{*}\right)
$$

is isometric for each $r \in \mathbb{N}$. To see this, it suffices to show that the correspond mapping

$$
T_{r} \stackrel{\wedge_{p}}{\otimes} T_{n} \stackrel{\wedge_{p}}{\otimes} V^{*} \rightarrow T_{r} \stackrel{\wedge_{p}}{\otimes} M_{n}(V)^{*}
$$

is isometric for each $r \in \mathbb{N}$. This is apparent from the commutative diagram

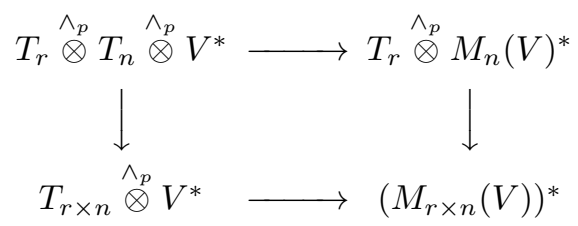

since we can obtain that the bottom and vertical mappings are isometric, we have $M_{n}(V)^{*} \cong T_{n}\left(V^{*}\right)$ is a p-completely isometric isomorphism.

We can obtain the p-completely isometric identifications

$$
T_{n}\left(\mathcal{N}_{p}(V, W)\right) \cong \mathcal{N}_{p}\left(V, T_{n}(W)\right) \cong \mathcal{N}_{p}\left(M_{n}(V), W\right)
$$

which is evident from the diagram

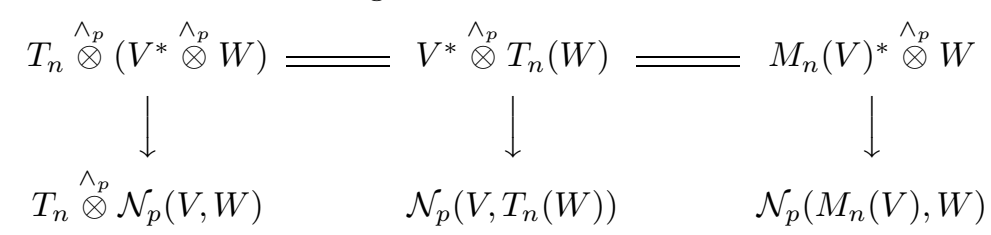

in which the column mappings are p-complete quotient mappings, and their null spaces are the same.

THEOREM 3.7. $\mathcal{N}_{p}$ is a p-operator space mapping ideal.

Proof. Let us suppose that we are given $\varphi \in M_{n}\left(\mathcal{N}_{p}(V, W)\right)$ and linear mappings $r: U \rightarrow V$ and $s: W \rightarrow X$. Since $\Phi: V^{*} \stackrel{\wedge_{p}}{\otimes} W \rightarrow V^{*} \stackrel{\vee_{p}}{\otimes} W$ is pcompletely contractive, we have $\|\varphi\|_{p c b} \leq \nu^{p}(\varphi)$. If we choose

$$
u \in M_{n}\left(V^{*} \stackrel{\wedge_{p}}{\otimes} W\right)
$$

with $\varphi=\Phi_{n}(u)$, it follows that

$$
s_{n} \circ \varphi \circ r=\Phi_{n}\left(u^{\prime}\right),
$$

where

$$
u^{\prime}=\left(r^{*} \otimes s\right)_{n}(u) \in M_{n}\left(U^{*} \stackrel{\wedge_{p}}{\otimes} X\right),
$$


and thus

$$
\nu_{n}^{p}\left(s_{n} \circ \varphi \circ r\right) \leq\left\|u^{\prime}\right\|_{\wedge_{p}} \leq\|s\|_{p c b}\|u\|_{\wedge_{p}}\|r\|_{p c b} .
$$

Taking the infimum over all $u$ with $\varphi=\Phi_{n}(u)$, we have that

$$
\nu_{n}^{p}\left(s_{n} \circ \varphi \circ r\right) \leq\|s\|_{p c b} \nu_{n}^{p}(\varphi)\|r\|_{p c b} .
$$

So we conclude that $\mathcal{N}_{p}$ is a p-operator space mapping ideal.

LEMMA 3.8. Let $V, W$ be $p$-operator spaces on $L_{p}$ spaces. If $\varphi^{*}: W^{*} \rightarrow V^{*}$ is a p-complete quotient mapping, then $\varphi: V \rightarrow W$ is a p-complete isometry.

Proof. By Lemma 4.6 in [2], the mapping $\varphi^{* *}: V^{* *} \rightarrow W^{* *}$ is p-completely isometric. We have a commutative diagram

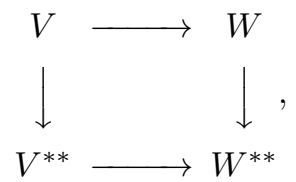

where the columns are p-completely isometric inclusions, and the bottom row is p-completely isometric. It follows that $\varphi: V \rightarrow W$ is p-completely isometric.

LEMmA 3.9. Let $V, W$ be p-operator spaces on $L_{p}$ spaces. Then the usual inclusion mapping $\iota: V \hookrightarrow V^{* *}$ induces the p-completely isometric injection $V \stackrel{\wedge_{p}}{\otimes} W \hookrightarrow V^{* *} \hat{\wedge}_{p} W$.

Proof. By Lemma 4.5 in [2], the mapping $\iota^{*}: V^{* * *} \rightarrow V^{*}$ is p-completely contractive.

For any $n \in \mathbb{N}$, the mapping $\varphi \rightarrow\left(\iota^{*}\right)_{n} \circ \varphi$ provides us with a quotient mapping in the top row of the diagram

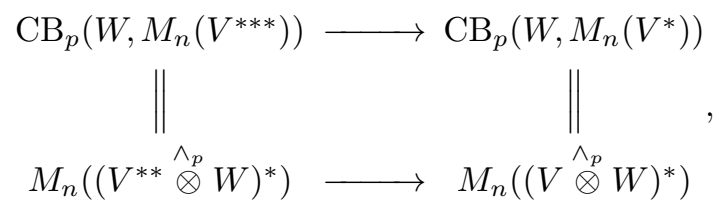

since we are given a p-complete contraction $\psi \in \mathrm{CB}_{p}\left(W, M_{n}\left(V^{*}\right)\right)$, then $\left(\iota_{V^{*}}\right)_{n} \circ \psi$ is the p-completely contractive preimage. Thus, the bottom row is also a quotient mapping. It follows that $\left(V^{* *} \stackrel{\wedge_{p}}{\otimes} W\right)^{*} \rightarrow\left(V \stackrel{\wedge_{p}}{\otimes} W\right)^{*}$ is a p-complete quotient mapping.

Owing to Lemma 3.8, we have that $V \stackrel{\wedge_{p}}{\otimes} W \hookrightarrow V^{* *} \stackrel{\wedge_{p}}{\otimes} W$ is p-completely isometric.

Proposition 3.10. Let $V, W$ be p-operator spaces on $L_{p}$ spaces and $\varphi$ : $V \rightarrow W$ is a $p$-completely bounded mapping, then $\varphi^{*}: W^{*} \rightarrow V^{*}$ satisfies

$$
\nu^{p}\left(\varphi^{*}\right) \leq \nu^{p}(\varphi) .
$$


If $V$ or $W$ is finite-dimensional, then $\nu^{p}\left(\varphi^{*}\right)=\nu^{p}(\varphi)$.

Proof. The result follows from Lemma 3.9 and a commutative diagram

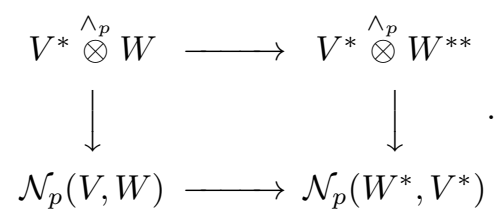

Proposition 3.11. Suppose that $L$ is a finite-dimensional p-operator space on $L_{p}$ space. Then for any p-operator space $W$ on $L_{p}$ space, the natural injection

$$
\mathcal{N}_{p}(L, W) \rightarrow \mathcal{N}_{p}\left(L, W^{* *}\right)
$$

is p-completely isometric.

Proof. The result follows from Lemma 3.9 and a commutative diagram

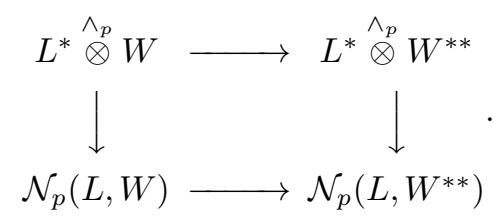

\section{P-completely integral mappings}

Definition 4.1. Let $V, W$ be p-operator spaces on $L_{p}$ spaces. We define the mapping $\varphi: V \rightarrow W$ with a p-operator space matrix norm $\iota^{p}(\cdot)$ to be p-completely integral, which

$\iota^{p}(\varphi)=\sup \left\{\nu^{p}\left(\varphi_{\mid L}\right):\right.$ for any finite-dimensional subspace $\left.L \subseteq V\right\}<\infty$.

And let $\mathcal{I}_{p}(V, W)$ denote the p-completely integral mapping spaces.

Given $\varphi \in M_{n}\left(\mathcal{I}_{p}(V, W)\right)$, we define

$\iota_{n}^{p}(\varphi)=\sup \left\{\nu_{n}^{p}\left(\varphi_{\mid L}\right):\right.$ for any finite-dimensional subspace $\left.L \subseteq V\right\}<\infty$.

Given a linear mapping $\varphi: V \rightarrow W$ and a finite-dimensional subspace $L$ of $V$ we have $\left.\varphi\right|_{L}=\varphi \circ r$, where $r: L \rightarrow V$ is the inclusion mapping, and thus

$$
\left\|\left.\varphi\right|_{L}\right\|_{p c b} \leq \nu^{p}\left(\left.\varphi\right|_{L}\right) \leq \nu^{p}(\varphi)\|r\|_{p c b}=\nu^{p}(\varphi) .
$$

From this, we infer that

$$
\|\varphi\|_{p c b} \leq \iota^{p}(\varphi) \leq \nu^{p}(\varphi)
$$

If $V$ is finite-dimensional, then from the definition $\nu^{p}(\varphi) \leq \iota^{p}(\varphi)$. So we have an isometric identification $\mathcal{I}_{p}(V, W) \cong \mathcal{N}_{p}(V, W)$.

THEOREM 4.2. $\mathcal{I}_{p}$ is a local p-operator space mapping ideal. 
Proof. To see this, let us suppose that we are given $\varphi \in M_{n}\left(\mathcal{I}_{p}(V, W)\right)$ and linear mappings $r: U \rightarrow V$ and $s: W \rightarrow X$. If $K$ is a finite-dimensional subspace of $U$ and we let $L=r(K)$, then

$$
\nu_{n}^{p}\left(\left.s_{n} \circ \varphi \circ r\right|_{K}\right) \leq\|s\|_{p c b} \nu_{n}^{p}\left(\left.\varphi\right|_{L}\right)\|r\|_{p c b} \leq\|s\|_{p c b} \iota_{n}^{p}(\varphi)\|r\|_{p c b},
$$

and thus

$$
\iota_{n}^{p}\left(s_{n} \circ \varphi \circ r\right) \leq\|s\|_{p c b} \iota_{n}^{p}(\varphi)\|r\|_{p c b} .
$$

Since $\|\varphi\|_{p c b} \leq \iota^{p}(\varphi)$, we have that $\mathcal{I}_{p}$ is a p-operator space mapping ideal. Then from Definition 4.1, we have that this p-mapping ideal is local.

Theorem 4.3. Let $V, W$ be p-operator spaces on $L_{p}$ spaces. The natural mapping $\mathcal{I}_{p}(V, W) \rightarrow \mathcal{I}_{p}\left(V, W^{* *}\right)$ is p-completely isometric.

Proof. Since $\mathcal{I}_{p}$ is a p-mapping ideal, this mapping is a p-complete contraction. On the other hand, letting $\lambda: W \rightarrow W^{* *}$ be the canonical injection, let us suppose that $\iota_{n}^{p}\left(\lambda_{n} \circ \varphi\right) \leq 1$. Given a finite-dimensional subspace $L \subseteq V$, it follows from Proposition 3.11 that

$$
\nu_{n}^{p}\left(\left.\varphi\right|_{L}\right)=\nu_{n}^{p}\left(\left.\lambda_{n} \circ \varphi\right|_{L}\right) \leq 1,
$$

and thus $\iota_{n}^{p}(\varphi) \leq 1$.

Let $V, W$ be p-operator spaces on $L_{p}$ spaces. We have a natural diagram of p-complete contractions

$$
\begin{aligned}
& \mathcal{N}_{p}\left(V, W^{*}\right) \subseteq \mathcal{I}_{p}\left(V, W^{*}\right) \subseteq \mathrm{CB}_{p}\left(V, W^{*}\right) \\
& V^{*} \stackrel{\stackrel{\AA \uparrow}{\otimes}}{\otimes} W^{*} \stackrel{\theta}{\rightarrow}\left(V \stackrel{\vee_{p}}{\otimes} W\right)^{*} \stackrel{\Phi^{*}}{\rightarrow}\left(V \stackrel{\downarrow_{p}}{\otimes} W\right)^{*},
\end{aligned}
$$

where $S: \mathrm{CB}_{p}\left(V, W^{*}\right) \cong\left(V \stackrel{\wedge_{p}}{\otimes} W\right)^{*}$ is a p-complete isometry determined by

$$
S(\varphi): V \otimes W \rightarrow \mathbb{C}: v \otimes w \mapsto \varphi(v)(w),
$$

$\Phi: V \stackrel{\wedge_{p}}{\otimes} W \rightarrow V \stackrel{\vee_{p}}{\otimes} W$ and $\widehat{\Phi}: V^{*} \stackrel{\wedge_{p}}{\otimes} W^{*} \rightarrow \mathcal{N}_{p}\left(V, W^{*}\right)$ are the canonical mappings. The map $\theta$ is determined by the fact that the bilinear mapping

$$
V^{*} \times W^{*} \rightarrow\left(V \stackrel{\wedge_{p}}{\otimes} W\right)^{*}
$$

is p-completely contractive in the sense that

$$
\|f \otimes g\| \leq\|f\|\|g\|
$$

for $f \in M_{r}\left(V^{*}\right)$ and $g \in M_{s}\left(W^{*}\right)$. The diagram commutes since it is immediate that $S(\widehat{\Phi}(F))=\Phi^{*}(\theta(F))$ for $F=f \otimes g\left(f \in V^{*}, g \in W^{*}\right)$, and extending linearly and using continuity, we find that this relation holds for all $F \in V^{*} \stackrel{\wedge_{p}}{\otimes} W^{*}$. 
LEMmA 4.4. Let $V, W$ be p-operator spaces on $L_{p}$ spaces. There is a $p$ completely contractive mapping

$$
S_{\text {int }}: \mathcal{I}_{p}\left(V, W^{*}\right) \rightarrow\left(V \stackrel{\vee_{p}}{\otimes} W\right)^{*}
$$

for which the following diagram commutes

$$
\begin{aligned}
& \mathcal{N}_{p}\left(V, W^{*}\right) \subseteq \mathcal{I}_{p}\left(V, W^{*}\right) \subseteq \mathrm{CB}_{p}\left(V, W^{*}\right)
\end{aligned}
$$

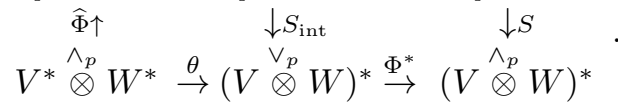

Proof. Our task is to show that for any $\phi \in M_{n}\left(\mathcal{I}_{p}\left(V, W^{*}\right)\right)$,

$$
S_{p}(\phi): V \otimes \vee_{p} W \rightarrow M_{n}
$$

satisfies $\left\|S_{n}(\phi)\right\|_{p c b} \leq \iota_{n}^{p}(\phi)$. From this, it will follow that the restriction $S_{\text {int }}$ of $S$ is p-completely contractive.

Given $\phi \in M_{n}\left(\mathrm{CB}_{p}\left(V, W^{*}\right)\right)$ with $\iota_{n}^{p}(\phi) \leq 1$, there is by definition a net $\psi_{\alpha}(v) \in M_{n}\left(W^{*}\right)$ converges to $\phi(v)$ in norm for all $v \in V$. It follows that the net of scalar matrices $\left(S_{n}\left(\psi_{\alpha}\right)\right)_{m}(u)$ converges to $\left(S_{n}(\phi)\right)_{m}(u)$ for any $u \in M_{m}(V \otimes W)$. Letting $\psi_{\alpha}=\widehat{\Phi}_{n}\left(F_{\alpha}\right)$ with $\left\|F_{\alpha}\right\|_{\wedge_{p}} \leq 1$, we have

$$
\left\|\left(S_{n}\left(\psi_{\alpha}\right)\right)_{m}(u)\right\|=\left\|\left(\theta_{n}\left(F_{\alpha}\right)\right)_{m}(u)\right\| \leq\left\|F_{\alpha}\right\|_{\wedge_{p}}\|u\|_{\vee_{p}}<\|u\|_{\vee_{p}} .
$$

Taking the limit, we see that $\left\|S_{n}(\phi)(u)\right\|_{p c b} \leq\|u\|_{\vee_{p}}$, and thus

$$
\left\|S_{n}(\phi)\right\|_{p c b} \leq 1 \text {. }
$$

Lemma 4.5. Let $V, W$ be p-operator spaces on $L_{p}$ spaces. Then the composition

$$
S_{0}: \mathcal{I}_{p}(V, W) \hookrightarrow \mathcal{I}_{p}\left(V, W^{* *}\right) \rightarrow\left(V \stackrel{\vee_{p}}{\otimes} W^{*}\right)^{*}
$$

is isometric.

Proof. By Theorem 4.3 and Lemma 4.4, we have the composition is contractive. Let us suppose that $\varphi \in \mathcal{I}_{p}(V, W)$ satisfies $\left\|S_{0}(\varphi)\right\| \leq 1$.

Since

$$
W^{*} \stackrel{\vee_{p}}{\otimes} V \cong V \stackrel{\vee_{p}}{\otimes} W^{*} \hookrightarrow \mathrm{CB}_{p}\left(W, V^{* *}\right) \cong\left(V^{*} \stackrel{\wedge p}{\otimes} W\right)^{*}
$$

are p-completely isometric, we may identify $W^{*} \stackrel{\vee_{p}}{\otimes} V$ with a p-operator subspace of $\left(V^{*} \stackrel{\wedge p}{\otimes} W\right)^{*}$. It follows from the Hahn-Banach theorem that $S_{0}(\varphi)$ has a contractive extension

$$
F_{\varphi} \in\left(V^{*} \stackrel{\wedge p}{\otimes} W\right)^{* *}
$$

From the bipolar theorem, we may choose a net of elements

$$
u_{\lambda} \in V^{*} \stackrel{\wedge p}{\otimes} W
$$


such that

$$
\left\|u_{\lambda}\right\|_{V^{*} \wedge{ }^{p} W}<1
$$

and $u_{\lambda}$ converges to $F_{\varphi}$ in the point-norm topology on $\left(V^{*} \stackrel{\wedge p}{\otimes} W\right)^{* *}$. It follows that

$$
\varphi_{\lambda}=\Phi\left(u_{\lambda}\right) \in \mathcal{N}_{p}(V, W)
$$

is a net with $\nu^{p}\left(\varphi_{\lambda}\right)<1$, and for each $v \in V$ and $g \in W^{*}$,

$$
\varphi_{\lambda}(v)(g)=u_{\lambda}(v \otimes g)=S_{0}(\varphi)(v \otimes g)=\varphi(v)(g) .
$$

Therefore, $\varphi_{\lambda}$ converges to $\varphi$ in the point-weak topology, and thus $\iota^{p}(\varphi) \leq 1$. We conclude that the composition is isometric.

THEOREM 4.6. If $L$ is a finite-dimensional p-operator space on $L_{p}$ space, then for any p-operator spaces on $L_{p}$ space $V$ we have the isometry

$$
S_{\text {int }}: \mathcal{I}_{p}\left(V, L^{*}\right) \cong\left(V \stackrel{\vee_{p}}{\otimes} L\right)^{*}
$$

Proof. It is immediate from Lemma 4.5.

Given p-operator spaces $V$ and $W$, Lee defined $V^{* *}: \stackrel{\vee_{p}}{\otimes}: W^{* *}, V \stackrel{\vee_{p}}{\otimes}: W^{* *}$ and $V^{* *}: \otimes W$, which were called the p-augmented, p-right augmented and p-left augmented injective tensor products, respectively (see [11]).

THEOREM 4.7. For any p-operator spaces $V$ and $W$ on $L_{p}$ spaces, the mapping

$$
S_{\text {int }}: \mathcal{I}_{p}\left(V, W^{*}\right) \rightarrow\left(V \stackrel{\vee_{p}}{\otimes} W\right)^{*}
$$

is an isometric surjection if and only if we have the natural isometric isomorphism

$$
V \stackrel{\vee_{p}}{\otimes}: W^{* *} \cong V \stackrel{\vee_{p}}{\otimes} W^{* *}
$$

Proof. Let us suppose that we have $V \stackrel{\vee_{p}}{\otimes}: W^{* *} \cong V \stackrel{\vee_{p}}{\otimes} W^{* *}$. For any

$$
\varphi \in \mathcal{I}_{p}\left(V, W^{*}\right)
$$

$F_{\varphi}=S_{\text {int }}(\varphi)=S(\varphi)$ is determined by $\left\langle F_{\varphi}, v \otimes w\right\rangle=\varphi(v)(w)$ (see Lemma 4.4). From Lemma 4.5 , we have the natural isometry

$$
S_{0}: \mathcal{I}_{p}\left(V, W^{*}\right) \hookrightarrow\left(V \stackrel{\vee_{p}}{\otimes} W^{* *}\right)^{*} .
$$

It follows that

$$
\begin{aligned}
\iota^{p}(\varphi) & =\sup \left\{\left|\left\langle F_{\varphi}, u\right\rangle\right|: u \in V \otimes W^{* *},\|u\|_{V \otimes V^{v_{p}}} \leq 1\right\} \\
& =\sup \left\{\left|\left\langle F_{\varphi}, u\right\rangle\right|: u \in V \otimes W^{* *},\|u\|_{V \otimes W^{* *}} \leq 1\right\} .
\end{aligned}
$$


Since the closed unit ball of $V \otimes \vee_{p} W$ is weak* dense in the closed unit ball of $\left(V \stackrel{\vee_{p}}{\otimes} W\right)^{* *}$,

$$
\iota^{p}(\varphi)=\sup \left\{\left|\left\langle F_{\varphi}, u\right\rangle\right|: u \in V \otimes W,\|u\|_{V \otimes \vee_{p}} \leq 1\right\}=\left\|F_{\varphi}\right\| .
$$

To prove that $S_{\text {int }}$ is a surjection, let us suppose that $f \in\left(V \stackrel{\vee_{p}}{\otimes} W\right)^{*}$. Then since the mapping $S: \mathrm{CB}_{p}\left(V, W^{*}\right) \cong\left(V \stackrel{\wedge_{p}}{\otimes} W\right)^{*}$ is a p-completely isometric surjection and $\Phi: V \stackrel{\wedge_{p}}{\otimes} W \rightarrow V \stackrel{\vee_{p}}{\otimes} W$ is contractive, there is a p-complete contraction $\varphi: V \rightarrow W^{*}$ such that $S(\varphi)=\Phi^{*}(f)$. Restricting to the algebraic tensor product $V \otimes W$, we have $F_{\varphi}=f$, and thus from the above calculations we obtain $\iota^{p}(\varphi)=\|f\|<\infty$. We conclude that $\varphi \in \mathcal{I}_{p}\left(V, W^{*}\right)$ and $S_{\text {int }}(\varphi)=f$.

Conversely, let us suppose that

$$
S_{\mathrm{int}}: \mathcal{I}_{p}\left(V, W^{*}\right) \rightarrow\left(V \stackrel{\vee_{p}}{\otimes} W\right)^{*}
$$

is an isometric surjection. Then we have the commutative diagram

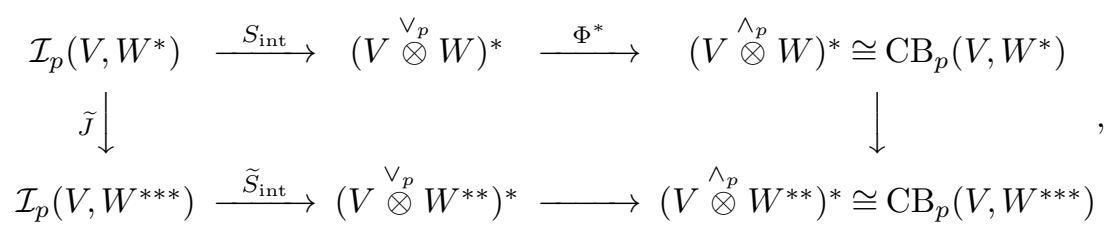

where $\widetilde{J}$ is the isometry described in Theorem 4.3, and the right column is the obvious isometric inclusion. Thus, if we let

$$
\eta=\widetilde{S}_{\text {int }} \circ \widetilde{J} \circ S_{\text {int }}^{-1},
$$

then we obtain a diagram of contractions

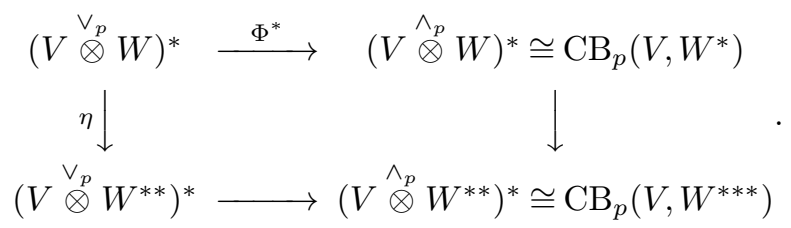

If we take the adjoints of the mappings in this diagram, then we obtain the commutative diagram

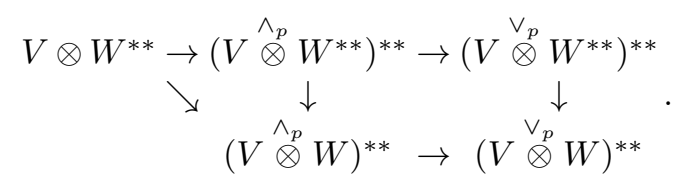

The bottom composition has range $V \otimes: \vee_{p} W^{* *}$. On the other hand, $V \otimes W^{* *}$ in $\left(V \otimes: \vee_{p} W^{* *}\right)^{* *}$, and thus the algebraic identification $V \otimes \vee_{p}$ $W^{* *}=V \otimes: \vee_{p} W^{* *}$ is an isometric isomorphism. 
The conditions $C_{p}, C_{p}^{\prime}$ and $C_{p}^{\prime \prime}$ of p-operator spaces on $L_{p}$ spaces have been studied by Lee (see [11]). Let $V$ be a p-operator space on $L_{p}$ space. We say $V$ satisfies condition $C_{p}$ if we have the isometry $V^{* *}: \stackrel{\vee_{p}}{\otimes} W^{* *} \cong V^{* *} \stackrel{\vee_{p}}{\otimes} W^{* *}$ for all p-operator spaces $W$ on $L_{p}$ spaces. It is equivalent to suppose that the isometry is a p-complete isometry, since Theorem 3.6 and the isometry imply that

$$
M_{n}\left(V^{* *}: \stackrel{\vee_{p}}{\otimes}: W^{* *}\right) \cong V^{* *}: \stackrel{\vee_{p}}{\otimes}: M_{n}(W)^{* *} \cong M_{n}\left(V^{* *} \stackrel{\vee_{p}}{\otimes} W^{* *}\right)
$$

Similarly, we say $V$ satisfies condition $C_{p}^{\prime}$ if we have the isometry $V \stackrel{\vee_{p}}{\otimes}: W^{* *} \cong$ $V \stackrel{\vee_{p}}{\otimes} W^{* *}$ for all p-operator spaces $W$ on $L_{p}$ spaces. We say $V$ satisfies condition $C_{p}^{\prime \prime}$ if we have the isometry $V^{* *}: \stackrel{\vee_{p}}{\otimes} \cong V^{* *} \vee_{p}^{\otimes} W$ for all p-operator spaces $W$ on $L_{p}$ spaces. Once again, these conditions are stable in the sense that if they hold, then these identifications are p-completely isometric isomorphisms.

Corollary 4.8. Let $V$ be a p-operator space on $L_{p}$ space.

(1) $V$ satisfies condition $C_{p}^{\prime}$ if and only $\mathcal{I}_{p}\left(V, W^{*}\right) \cong\left(V \stackrel{\vee_{p}}{\otimes} W\right)^{*}$ is an isometry for all p-operator spaces $W$ on $L_{p}$ spaces;

(2) $V$ satisfies condition $C_{p}^{\prime \prime}$ if and only if $\mathcal{I}_{p}\left(W, V^{*}\right) \cong\left(V \stackrel{\vee_{p}}{\otimes} W\right)^{*}$ is an isometry for all p-operator spaces $W$ on $L_{p}$ spaces.

Proof. This is an immediate consequence of Theorem 4.7 and the definitions of the conditions $C_{p}^{\prime}$ and $C_{p}^{\prime \prime}$.

\section{P-completely 1 -summing and $\infty$-summing mappings}

Completely 1-summing mappings have been studied by Effros and Ruan [6] and completely $\infty$-summing mappings have been considered by Dong [3]. In this section, we will define and study p-completely 1 -summing and $\infty$ summing mappings.

Definition 5.1. If $\varphi: V \rightarrow W$ is a linear mapping of p-operator spaces on $L_{p}$ spaces, then we define $\pi_{1}^{p}(\varphi)$ in $[0, \infty]$ by

$$
\begin{aligned}
\pi_{1}^{p}(\varphi) & =\left\|\operatorname{id}_{T_{\infty}} \otimes \varphi: T_{\infty} \stackrel{\vee_{p}}{\otimes} V \rightarrow T_{\infty} \stackrel{\wedge p}{\otimes} W\right\| \\
& =\sup \left\{\left\|\operatorname{id}_{T_{r}} \otimes \varphi: T_{r} \stackrel{\vee_{p}}{\otimes} V \rightarrow T_{r} \stackrel{\wedge p}{\otimes} W\right\|: r \in \mathbb{N}\right\} .
\end{aligned}
$$

If $\pi_{1}^{p}(\varphi)<\infty$, we say that $\varphi$ is p-completely 1 -summing and we refer to $\pi_{1}^{p}(\varphi)$ as the p-completely 1 -summing norm of $\varphi$. We let $\Pi_{1}^{p}(V, W)$ denote the space of all p-completely 1-summing mappings from $V$ into $W$.

THEOREM 5.2. For any p-operator spaces on $L_{p}$ spaces $V$ and $W$, a linear mapping $\varphi: V \rightarrow W$ satisfies $\pi_{1}^{p}(\varphi)<1$ if and only if for each $n \in \mathbb{N}$ and p-complete contraction $\theta: M_{n} \rightarrow V, \nu^{p}(\varphi \circ \theta) \leq 1$. 
Proof. This is apparent from the commutative diagram

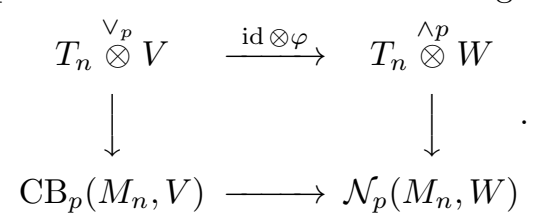

Corollary 5.3. Let $V$ and $W$ be p-operator spaces on $L_{p}$ spaces. The bifunctor $\Pi_{1}^{p}:(V, W) \mapsto\left(\Pi_{1}^{p}(V, W), \Pi_{1}^{p}\right)$ is a local p-operator space mapping ideal, and for any linear mapping $\varphi: V \rightarrow W, \pi_{1}^{p}(\varphi) \leq \iota^{p}(\varphi)$.

Proof. If $r=1$, we have $\left\|\mathrm{id}_{T_{r}}: T_{r} \stackrel{\vee_{p}}{\otimes} V \rightarrow T_{r} \stackrel{\wedge p}{\otimes} W\right\|$. Then we have $\|\varphi\| \leq \pi_{1}^{p}(\varphi)$. Suppose linear mappings $r: U \rightarrow V$ and $s: W \rightarrow X$. Then it is apparent from the diagram

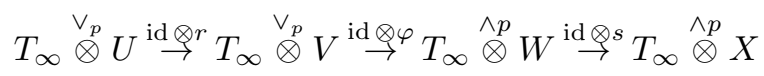

that

$$
\pi_{1}^{p}(s \circ \varphi \circ r) \leq\|s\| \pi_{1}^{p}(\varphi)\|r\|
$$

Therefore $\Pi_{1}^{p}$ is a p-mapping ideal. Since $\Pi_{1}^{p}$ has the p-ideal property, it is clear that for every finite-dimensional p-operator subspace $L \subseteq V$,

$$
\pi_{1}^{p}\left(\left.\varphi\right|_{L}\right) \leq \pi_{1}^{p}(\varphi)
$$

On the other hand, suppose that for any finite-dimensional p-operator subspace $L \subseteq V, \pi_{1}^{p}\left(\left.\varphi\right|_{L}\right) \leq 1$. For any $n \in \mathbb{N}$ and p-complete contraction $\psi: M_{n} \rightarrow V$, we set $L=\psi\left(M_{n}\right)$. Since $\pi_{1}^{p}\left(\left.\varphi\right|_{L}\right) \leq 1$, it follows from Theorem 5.2 that

$$
\nu^{p}(\varphi \circ \psi)=\nu^{p}\left(\left.\varphi\right|_{L} \circ \psi\right) \leq 1
$$

Theorem 5.2 shows that $\pi_{1}^{p}(\varphi) \leq 1$ and therefore $\Pi_{1}^{p}$ is local.

If $\nu^{p}(\varphi) \leq 1$, then for any $n \in \mathbb{N}$ and each p-complete contraction $\psi: M_{n} \rightarrow V$

$$
\nu^{p}(\varphi \circ \psi) \leq \nu^{p}(\varphi) \cdot\|\psi\|_{p c b} \leq 1
$$

and from Theorem 5.2,

Since $\Pi_{1}^{p}$ and $\mathcal{I}_{p}$ are local,

$$
\pi_{1}^{p}(\varphi) \leq \nu^{p}(\varphi)
$$

$$
\begin{aligned}
\pi_{1}^{p}(\varphi) & =\sup \left\{\pi_{1}^{p}\left(\left.\varphi\right|_{L}\right): \text { for any finite-dimensional subspace } L \subseteq V\right\} \\
& \leq \sup \left\{\nu^{p}\left(\left.\varphi\right|_{L}\right): \text { for any finite-dimensional subspace } L \subseteq V\right\} \\
& =\iota^{p}(\varphi) .
\end{aligned}
$$

Definition 5.4. If $\varphi: V \rightarrow W$ is a linear mapping of p-operator spaces on $L_{p}$ spaces, then we define $\pi_{\infty}^{p}(\varphi)$ in $[0, \infty]$ by

$$
\begin{aligned}
\pi_{\infty}^{p}(\varphi) & =\left\|\operatorname{id}_{M_{\infty}} \otimes \varphi: M_{\infty} \stackrel{\vee_{p}}{\otimes} V \rightarrow M_{\infty} \stackrel{\wedge p}{\otimes} W\right\| \\
& =\sup \left\{\left\|\operatorname{id}_{M_{r}} \otimes \varphi: M_{r} \stackrel{\vee_{p}}{\otimes} V \rightarrow M_{r} \stackrel{\wedge p}{\otimes} W\right\|: r \in \mathbb{N}\right\} .
\end{aligned}
$$


This definition is 'stable' in the sense that we may replace the bounded norms with p-completely bounded norms. To see this, let us suppose that $\pi_{\infty}^{p}(\varphi) \leq 1$. Let us fix $r$. We have

$$
\begin{aligned}
\left\|\operatorname{id}_{M_{r}} \otimes \varphi\right\|_{p c b}= & \sup \left\{\| \operatorname{id}_{M_{n}} \otimes \operatorname{id}_{M_{r}} \otimes \varphi:\right. \\
& \left.M_{n} \stackrel{\vee_{p}}{\otimes}\left(M_{r} \stackrel{\vee_{p}}{\otimes} V\right) \rightarrow M_{n} \stackrel{\vee_{p}}{\otimes}\left(M_{r} \stackrel{\wedge p}{\otimes} W\right) \|: n \in \mathbb{N}\right\} .
\end{aligned}
$$

From Theorem 2.1 and the definition of $\pi_{\infty}^{p}$, the two mappings in the diagram

$$
\begin{aligned}
M_{n} \stackrel{\vee_{p}}{\otimes}\left(M_{r} \stackrel{\vee_{p}}{\otimes} V\right) & =M_{n r} \stackrel{\vee_{p}}{\otimes} V \rightarrow M_{n r} \stackrel{\wedge p}{\otimes} W \\
& =\left(M_{n} \stackrel{\vee_{p}}{\otimes} M_{r}\right) \stackrel{\wedge p}{\otimes} W \rightarrow M_{n} \stackrel{\vee_{p}}{\otimes}\left(M_{r} \stackrel{\wedge p}{\otimes} W\right)
\end{aligned}
$$

are contractions, and thus $\left\|\operatorname{id}_{M_{r}} \otimes \varphi\right\|_{p c b} \leq 1$. If we let $r=1$, then $\|\varphi\|_{p c b} \leq 1$, and thus $\|\varphi\|_{p c b} \leq \pi_{\infty}^{p}(\varphi)$. If $\pi_{\infty}^{p}(\varphi)<\infty$, we say that $\varphi$ is p-completely $\infty$ summing and we refer to $\pi_{\infty}^{p}(\varphi)$ as the p-completely $\infty$-summing norm of $\varphi$. We let $\Pi_{\infty}^{p}(V, W)$ denote the space of all p-completely $\infty$-summing mappings from $V$ into $W$.

THEOREM 5.5. For any p-operator spaces $V$ and $W$ on $L_{p}$ spaces, a linear mapping $\varphi: V \rightarrow W$ satisfies $\pi_{\infty}^{p}(\varphi)<1$ if and only if for each $n \in \mathbb{N}$ and $p$-complete contraction $\theta: T_{n} \rightarrow V, \nu^{p}(\varphi \circ \theta) \leq 1$.

Proof. This is apparent from the commutative diagram

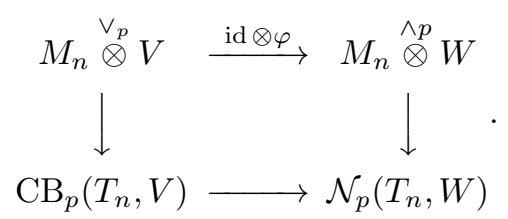

Corollary 5.6. Let $V$ and $W$ be p-operator spaces on $L_{p}$ spaces. The bifunctor $\Pi_{\infty}^{p}:(V, W) \mapsto\left(\Pi_{\infty}^{p}(V, W), \Pi_{\infty}^{p}\right)$ is a local p-operator space mapping ideal, and for any linear mapping $\varphi: V \rightarrow W, \pi_{\infty}^{p}(\varphi) \leq \iota^{p}(\varphi)$.

Proof. We may use the argument for the p-completely 1-summing norm.

THEOREM 5.7. Given p-operator spaces on $L_{p}$ spaces $V, W$ and a linear mapping $\varphi: V \rightarrow W$, we have $\pi_{1}^{p}(\varphi) \leq \pi_{\infty}^{p}\left(\varphi^{*}\right)$. Moreover, we have $\pi_{1}^{p}(\varphi)=\pi_{\infty}^{p}\left(\varphi^{*}\right)$ for any $p$-operator space $W$ and linear mapping $\varphi: V \rightarrow W$ if and only if $\mathcal{I}_{p}\left(V, M_{n}\right)=\mathcal{N}_{p}\left(V, M_{n}\right)$ for any $n \in \mathbb{N}$.

Proof. Since $M_{n} \stackrel{\wedge p}{\otimes} V^{*} \rightarrow\left(T_{n} \stackrel{\vee_{p}}{\otimes} V\right)^{*}$ is norm-decreasing, we conclude that

$$
\begin{aligned}
\pi_{1}^{p}(\varphi) & =\sup \left\{\left\|\operatorname{id}_{T_{n}} \otimes \varphi: T_{n} \stackrel{\vee_{p}}{\otimes} V \rightarrow T_{n} \stackrel{\wedge p}{\otimes} W\right\|: n \in \mathbb{N}\right\} \\
& =\sup \left\{\left\|\left(\operatorname{id}_{T_{n}} \otimes \varphi\right)^{*}:\left(T_{n} \stackrel{\wedge p}{\otimes} W\right)^{*} \rightarrow\left(T_{n} \stackrel{\vee}{\otimes} V\right)^{*}\right\|: n \in \mathbb{N}\right\}
\end{aligned}
$$




$$
\begin{aligned}
& \leq \sup \left\{\left\|\operatorname{id}_{M_{n}} \otimes \varphi^{*}: M_{n} \stackrel{\vee_{p}}{\otimes} W^{*} \rightarrow M_{n} \stackrel{\wedge p}{\otimes} V^{*}\right\|: n \in \mathbb{N}\right\} \\
& =\pi_{\infty}^{p}\left(\varphi^{*}\right) .
\end{aligned}
$$

If $\mathcal{I}_{p}\left(V, M_{n}\right)=\mathcal{N}_{p}\left(V, M_{n}\right)$, then $M_{n} \stackrel{\wedge p}{\otimes} V^{*} \rightarrow\left(T_{n} \stackrel{\vee_{p}}{\otimes} V\right)^{*}$ is isometric, and the above calculation implies that $\pi_{1}^{p}(\varphi)=\pi_{\infty}^{p}\left(\varphi^{*}\right)$.

Conversely, we first prove $\Pi_{\infty}^{p}\left(T_{n}, V^{*}\right)=\mathcal{N}_{p}\left(T_{n}, V^{*}\right)$. In fact, it follows from Corollary 5.6 that $\pi_{\infty}^{p}(\psi) \leq \iota^{p}(\psi) \leq \nu^{p}(\psi)$ for any $\psi: T_{n} \rightarrow V^{*}$. Suppose that $\pi_{\infty}^{p}(\psi) \leq 1$ for any $\psi: T_{n} \rightarrow V^{*}$. Theorem 5.5 shows that for $\operatorname{id}_{T_{n}}: T_{n} \rightarrow T_{n}$

$$
\nu^{p}(\psi)=\nu^{p}\left(\psi \circ \operatorname{id}_{T_{n}}\right) \leq 1 .
$$

Therefore, $\nu^{p}(\psi)=\pi_{\infty}^{p}(\psi)$ and $\Pi_{\infty}^{p}\left(T_{n}, V^{*}\right)=\mathcal{N}_{p}\left(T_{n}, V^{*}\right)$.

Thus we have the isometries

$$
\Pi_{1}^{p}\left(V, M_{n}\right)=\Pi_{\infty}^{p}\left(T_{n}, V^{*}\right)=\mathcal{N}_{p}\left(T_{n}, V^{*}\right)=\mathcal{N}_{p}\left(V, M_{n}\right),
$$

where the first equation follows from the hypothesis and the third from Proposition 3.10. Then, it follows from Corollary 5.6 we easily have

$$
\Pi_{1}^{p}\left(V, M_{n}\right)=\mathcal{I}_{p}\left(V, M_{n}\right)=\mathcal{N}_{p}\left(V, M_{n}\right)
$$

\section{P-local reflexivity}

Definition 6.1. We say that a p-operator space $W$ on $L_{p}$ space is p-locally reflexive if for any finite-dimensional p-operator space $L$ on $L_{p}$ space, every p-complete contraction $\varphi: L \rightarrow W^{* *}$ is the point-weak* limit of a net of linear mappings $\varphi_{\alpha}: L \rightarrow W$ with $\left\|\varphi_{\alpha}\right\|_{p c b} \leq 1$.

THEOREM 6.2. Suppose that $W$ is a p-operator space on $L_{p}$ space. Then the following are equivalent:

(1) $W$ is p-locally reflexive;

(2) For any finite-dimensional p-operator space $L$ on $L_{p}$ space, we have the isometry

$$
L^{*} \stackrel{\wedge_{p}}{\otimes} W^{*} \cong\left(L \stackrel{\vee_{p}}{\otimes} W\right)^{*}
$$

$(2)^{\prime}$ For any finite-dimensional p-operator space $L$ on $L_{p}$ space, we have the isometry

$$
\mathcal{I}_{p}\left(W, L^{*}\right) \cong \mathcal{N}_{p}\left(W, L^{*}\right)
$$

(3) For any p-operator space $V$ on $L_{p}$ space, we have the isometry

$$
\mathcal{I}_{p}\left(V, W^{*}\right) \cong\left(V \stackrel{\vee_{p}}{\otimes} W\right)^{*}
$$

(4) W satisfies condition $C_{p}^{\prime \prime}$. 
Proof. We have already proved $(3) \Leftrightarrow(4)$ (see Corollary 4.8 ).

$(2) \Leftrightarrow(2)^{\prime}$ It is immediate from Theorem 4.6 .

$(1) \Leftrightarrow(2)$ Since for any finite-dimensional p-operator space $L$ on $L_{p}$ space,

$$
\left(L^{*} \stackrel{\wedge_{p}}{\otimes} W^{*}\right)^{*} \cong \mathrm{CB}_{p}\left(L^{*}, W^{* *}\right) \cong L \stackrel{\vee_{p}}{\otimes} W^{* *},
$$

(2) holds if and only if we have the natural isometric isomorphism

$$
L \stackrel{\vee_{p}}{\otimes} W^{* *} \cong\left(L \stackrel{\vee_{p}}{\otimes} W\right)^{* *} .
$$

The corresponding is explicitly given by the norm-increasing linear isomorphism

$$
\tau: L \stackrel{\vee_{p}}{\otimes} W^{* *} \rightarrow\left(L \stackrel{\vee_{p}}{\otimes} W\right)^{* *} .
$$

Thus, the relation is isometric if and only if

$$
\varphi \in\left(L \stackrel{\vee_{p}}{\otimes} W^{* *}\right)_{\|\cdot\| \leq 1} \cong \mathrm{CB}_{p}\left(L^{*}, W^{* *}\right)_{\|\cdot\|_{p c b} \leq 1}
$$

implies that

$$
\varphi \in\left(L \stackrel{\vee_{p}}{\otimes} W\right)_{\|\cdot\| \leq 1}^{* *}
$$

From the bipolar theorem, the latter is the case if and only if $\varphi$ is a weak* limit of elements in

$$
\left(L \stackrel{\vee_{p}}{\otimes} W\right)_{\|\cdot\| \leq 1} \cong \mathrm{CB}_{p}\left(L^{*}, W\right)_{\|\cdot\|_{p c b} \leq 1} .
$$

Since it is evident that

$$
\tau: \mathrm{CB}_{p}\left(L^{*}, W^{* *}\right) \rightarrow\left(L \stackrel{\vee_{p}}{\otimes} W\right)^{* *}
$$

is a homeomorphism in the point-weak* and weak* topologies, we are done.

$(3) \Rightarrow(2)$ For any finite-dimensional p-operator space $L$ on $L_{p}$ space, we have the isometries

$$
L^{*} \stackrel{\wedge_{p}}{\otimes} W^{*} \cong \mathcal{N}_{p}\left(L, W^{*}\right) \cong \mathcal{I}_{p}\left(L^{*}, W^{*}\right) \cong\left(L \stackrel{\vee_{p}}{\otimes} W\right)^{*}
$$

$(2) \Rightarrow(3)$ From Lemma 4.4, we have seen that

$$
S_{\mathrm{int}}: \mathcal{I}_{p}\left(V, W^{*}\right) \rightarrow\left(V \stackrel{\vee_{p}}{\otimes} W\right)^{*}
$$

is a contractive injection. Let us suppose that the mapping in (2) is isometric. If we have a contractive functional $F \in\left(V \stackrel{\vee_{p}}{\otimes} W\right)^{*}$, then $F=S(\varphi)$ for some $\varphi: V \rightarrow W^{*}$ (see Lemma 4.4). For any finite-dimensional subspace $L \subseteq V$ and p-complete contraction $\psi: L \rightarrow V$, we have

$$
F \circ\left(\psi \otimes \operatorname{id}_{W}\right) \in\left(V \stackrel{\vee_{p}}{\otimes} W\right)^{*} \quad \text { and } \quad \varphi \circ \psi: L \rightarrow W^{*} .
$$

Since for any $x \in L, y \in W$

$$
\left(F \circ\left(\psi \otimes \operatorname{id}_{W}\right)\right)(x \otimes y)=F(\psi(x) \otimes y)=\varphi(\psi(x))(y),
$$


we have $F \circ\left(\psi \otimes \operatorname{id}_{W}\right)=S(\varphi \circ \psi)$. Thus from $(2)$ and $L^{*} \stackrel{\wedge_{p}}{\otimes} W^{*} \cong \mathcal{N}_{p}\left(L, W^{*}\right)$,

$$
\nu^{p}(\varphi \circ \psi)=\left\|F \circ\left(\psi \otimes \operatorname{id}_{W}\right)\right\| \leq\|F\| .
$$

From the definition of $\iota^{p}(\varphi)$, we have $\iota^{p}(\varphi)_{\vee_{p}} \leq\|F\|$. Therefore, $\iota^{p}(\varphi)=\|F\|$ for $\varphi \in \mathcal{I}_{p}\left(V, W^{*}\right)$ and thus $\mathcal{I}_{p}\left(V, W^{*}\right) \cong\left(V \otimes \otimes^{p} W\right)^{*}$.

Corollary 6.3. Suppose that $W$ is a p-operator space on $L_{p}$ space. If $W$ is $p$-locally reflexive, then any subspace $X \subseteq W$ is $p$-locally reflexive.

Proof. For any finite-dimensional p-operator space $L$ on $L_{p}$ space, from Theorem 6.2, we have the isometry

$$
L^{*} \stackrel{\wedge_{p}}{\otimes} W^{*} \cong\left(L \stackrel{\vee_{p}}{\otimes} W\right)^{*}
$$

Since

$$
\left(L^{*} \stackrel{\wedge_{p}}{\otimes} W^{*}\right)^{*} \cong \mathrm{CB}_{p}\left(L^{*}, W^{* *}\right) \cong L \stackrel{\vee_{p}}{\otimes} W^{* *}
$$

$L^{*} \stackrel{\wedge_{p}}{\otimes} W^{*} \cong\left(L \stackrel{\vee_{p}}{\otimes} W\right)^{*}$ holds if and only if we have the natural isometric isomorphism

$$
L \stackrel{\vee_{p}}{\otimes} W^{* *} \cong\left(L \stackrel{\vee_{p}}{\otimes} W\right)^{* *} .
$$

Then $X$ is p-locally reflexive from Theorem 6.2 and the commutative diagram

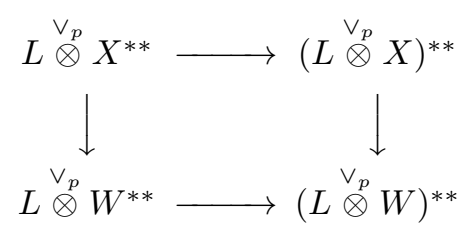

in which the columns are isometric.

Corollary 6.4. Suppose that $W$ is a p-operator space on $L_{p}$ space. If $W$ is p-locally reflexive, then $\Pi_{1}^{p}(W, V) \cong \Pi_{\infty}^{p}\left(V^{*}, W^{*}\right)$ for any p-operator space $V$ on $L_{p}$ space and linear mapping $\varphi: W \rightarrow V$.

Proof. It follows from Theorem 5.7 and Theorem 6.2 immediately.

Acknowledgment. The authors give their sincere thanks to the referee and Professor Z.-J. Ruan for their valuable comments and improvements.

\section{REFERENCES}

[1] G. An, J.-J. Lee and Z.-J. Ruan, On p-approximation properties for $p$-operator spaces, J. Funct. Anal. 259 (2010), 933-974. MR 2652178

[2] M. Daws, P-operator spaces and Figà-Talamanca-Herz algebras, J. Operator Theory 63 (2010), 47-83. MR 2606882

[3] Z. Dong, The OLLP and $\tau$-local reflexivity of operator spaces, Illinois J. Math. 51 (2007), 1103-1122. MR 2417418 
[4] E. G. Effros, M. Junge and Z.-J. Ruan, Intergral mappings and the principle of local reflexivity for non-commutative $L^{1}$ space, Ann. of Math. (2) 151 (2000), 59-92. MR 1745018

[5] E. G. Effros and Z.-J. Ruan, Mapping spaces and liftings for operator spaces, Proc. Lond. Math. Soc. (3) 69 (1994), 171-197. MR 1272425

[6] E. G. Effros and Z.-J. Ruan, The Grothendieck-Pietsh and Dvoretzky-Rogers theorems for operator spaces, J. Funct. Anal. 69 (1994), 171-197. MR 1276165

[7] E. G. Effros and Z.-J. Ruan, On the analogues of integral mappings and local reflexivity for operator spaces, Indiana Univ. Math. J. 46 (1997), 1289-1310. MR 1631592

[8] E. G. Effros and Z.-J. Ruan, Operator spaces, London Mathematical Society Monographs. New Series, vol. 23, Oxford University Press, New York, 2000. MR 1793753

[9] C. Le Merdy, Factorization of p-completely bounded multilinear maps, Pacific J. Math. 172 (1996), 187-213. MR 1379292

[10] J.-J. Lee, On p-operator spaces and their applications, Ph.D. thesis, University of Illinois (Urbana-Champaign), 2010. MR 2873498

[11] J.-J. Lee, Conditions $C_{p}, C_{p}^{\prime}$, and $C_{p}^{\prime \prime}$ for p-operator spaces, preprint, 2012; available at arXiv:1209.1864.

[12] J.-J. Lee, Hahn-Banach type extension theorems on p-operator spaces, preprint, 2013; available at arXiv:1303.3513.

[13] G. Pisier, Completely bounded maps between sets of Banach space operators, Indiana Univ. Math. J. 39 (1990), 249-277. MR 1052019

Y. F. Zhao, Department of Mathematics, Zhejiang University, Hangzhou 310027 , P.R. CHINA

Current address: Department of Mathematics, Zhejiang International Studies University, Hangzhou 310012, P.R. China

E-mail address: zhaoyafei_zju@163.com

Z. Dong, Department of Mathematics, Zhejiang University, Hangzhou 310027 , P.R. CHINA

E-mail address: dongzhe@zju.edu.cn 